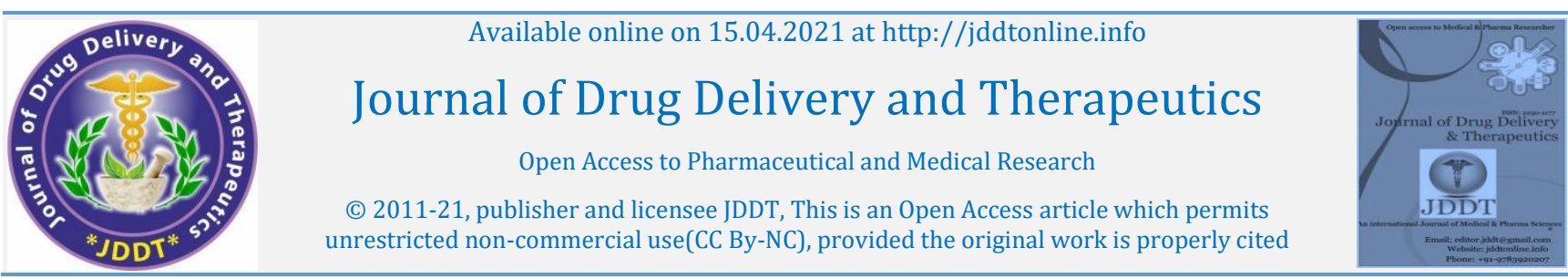

(C) 2011-21, publisher and licensee JDDT, This is an Open Access article which permits
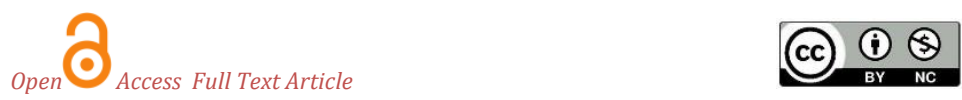

Research Paper

\title{
Comparative Study of Different Products of Carbamazepine Tablets Available in Iraqi Market
}

\author{
Mohammed K. Al-Shaheen, Saad M. Majeed, and Radhwan N. Al-Zidan* \\ Department of Pharmaceutics, College of Pharmacy, University of Mosul, Iraq
}

\section{Article Info:

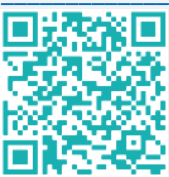 \\ Article History: \\ Received 13 Feb 2021 \\ Review Completed 23 March 2021 \\ Accepted 29 March 2021 \\ Available online 15 April 2021}

Cite this article as:

Al-Shaheen MK, Majeed, SM, Al-Zidan RN, Comparative Study of Different Products of Carbamazepine Tablets Available in Iraqi Market, Journal of Drug Delivery and Therapeutics. 2021; 11(2-s):50-55

DOI: http://dx.doi.org/10.22270/jddt.v11i2-s.4808

*Address for Correspondence:

Radhwan N. Al-Zidan, Department of Pharmaceutics, College of Pharmacy, University of Mosul, Iraq

\begin{abstract}
Carbamazepine (CBZ) is a widely used antiepileptic drug to control grand mal epilepsy, as well as for the treatment of peripheral neuralgia. According to the biopharmaceutical classification system (BCS), CBZ is considered a class II drug. CBZ is characterized by a slow and irregular gastrointestinal absorption, with irregular oral bioavailability; due to its low water solubility. Therefore, the release of the drug from the dosage form (tablets in this study) and the subsequent step of dissolution represent the most important parameters that decide whether a sufficient plasma concentration will be achieved or not. In the current study, the FTIR study for the pure API, CBZ, and the different commercially available brands of CBZ conventional tablets, available in the Iraqi drug market (Mosul city as an example), were examined. Subsequently, various quality control parameters such as the weight variation, content uniformity, friability, and hardness of the conventional CBZ tablets were conducted. Moreover, the disintegration and the dissolution tests of the different brands of CBZ available in the Iraqi drug market were performed.
\end{abstract}

Keywords: Antiepileptic Drug, Bioequivalence; Epilepsy; Generic; Brand vs generic; IVIVC; QC

\section{INTRODUCTION}

During large-scale drug manufacturing, in-process quality control should be conducted to prevent the production and flow of substandard pharmaceutical products into the market. Quality thus can be defined as the suitability of the goods or service to the determining qualifications. ${ }^{1}$

Carbamazepine (CBZ), a class II drug, is a widely used antiepileptic drug, usually prescribed to control tonic-clonic seizures in addition to other medical conditions like peripheral neuralgia. CBZ is characterized by slow and irregular gastrointestinal absorption due to its low water solubility. ${ }^{2}$

CBZ is available in the Iraqi drug market in different dosage forms including suspension, conventional tablets, controlledrelease, and extended-release tablets that belong to different pharmaceutical companies. Following oral administration, suspensions are absorbed slightly faster than conventional tablets. $^{3}$

In this study, conventional CBZ tablets were subjected to various tests, specified by the US pharmacopeia, to ensure the compatibility of the carbamazepine tablets with the required quality standards. The conducted tests included weight variation, content uniformity, hardness, friability, disintegration, and dissolution tests. Additionally, FTIR studies for the pure material as well as for the tested brands were conducted to prove the purity and seek any possible drug-excipient interaction within the dosage form. ${ }^{4}$ Manufacturers may follow different knowhows, add different excipients, use different methods of preparation, and variable pre- and post-compression parameters. So, different drug release profiles are expected. ${ }^{5}$ Hence, the drug release profiles of the selected brands were measured and compared.

\section{MATERIALS AND METHOD}

\section{Material}

Carbamazepine pure powder was a kind gift sample from AwaMedica Co. Pharmaceutical company, Iraq. In this study, four generic products of commercial conventional tablets containing $200 \mathrm{mg}$ of CBZ were used and coded as CBZ A (Carbatol 200, manufactured by Dar Al Dawa, Jordan, Lot: 05EY); CBZ B (®Tegretol 200, manufactured by Novartis, Turkey; Lot KL795); CBZ C (CarbaZepineAwa 200, manufactured by AwaMedica Co., Iraq; Lot PC 2065); and CBZ D (Tegretol® 200, manufactured by Novartis Pharma, Italy; Lot TCY 93).

\section{Methods}

\section{Preparation of standard calibration curve for carbamazepine}

In order to determine the standard calibration curve for CBZ, a stock solution containing $200 \mathrm{mcg} / \mathrm{ml}$ was prepared in distilled water containing $0.5 \% \mathrm{w} / \mathrm{v}$ sodium lauryl sulfate (NaLS). Sonicated for 30 minutes, then dilutions were made 
to prepare a series of solutions containing CBZ in different concentrations. In these solutions absorbance values at 285 nm were determined using Cary UV-VIS spectrophotometer. Subsequently, by plotting the concentration values ( $\mathrm{x}$ ) versus absorbances (y) a calibration curve of CBZ in distilled water containing $0.5 \%$ NaLS was determined. Analytical parameters for the assay of $\mathrm{CBZ}$ were calculated by the ANOVA test.

\section{FTIR Analytical study}

FT-IR study on the pure CBZ powder as well as for each brand was done using Bruker-Alpha ATR-FTIR spectrophotometer (Germany).

\section{Weight variation test}

Each tablet $(n=20)$ belonging to each manufacturer was weighed with an electric balance (ADAM AFA120LC), the average weight was calculated for each brand then deviations from the average were calculated according to permissible percentage allowed in USP. ${ }^{6}$

\section{Hardness test}

This test was applied with a hardness tester (SaintyCo hardness tester) on 10 tablets for each brand and the result was expressed as an average. 6

\section{Friability test}

Double drum Roche Friabilitor was used to determine the friability for different brands involved in this study, 20 tablets from each brand were selected randomly, weighed, and put into the friabilitor. Tablets were rotated at $25 \mathrm{rpm}$, then after 100 rotations the friability percentage was calculated for each product. 6

\section{Content uniformity test}

The amount of CBZ in tablets from each brand was determined according to the method described in the USP Pharmacopeia. ${ }^{6} 10$ tablets from each brand were randomly selected, weighed individually and the average weight is then determined, then the tablets were crushed and an aliquot weight equivalent to $200 \mathrm{mg} \mathrm{CBZ}$ is carefully determined and dissolved in a $1000 \mathrm{ml}$ volumetric flask containing $0.5 \% \mathrm{w} / \mathrm{v}$ NaLS in distilled water, sonicated for 15 minutes and filtered, the filtrate is scanned at a wavelength equal to $285 \mathrm{~nm}$ using Carry UV/VIS double beam spectrophotometer and the concentration of the filtrate was determined from the calibration curve equation, then the results were expressed as a percentage of drug content. 6

\section{Disintegration test}

6 tablets of each brand were placed in a medium of $0.1 \mathrm{~B} \mathrm{HCl}$ $900 \mathrm{ml}$ at $37{ }^{\circ} \mathrm{C}$ in the cylindrical tubes of the basket of disintegration apparatus (SantyCo BJ-2) and testing for measuring disintegration time as the time at which no particles remained in the basket of the system and passed out the bottom mesh of the tube was recorded as the disintegration time. The mean disintegration time was calculated for each batch. 6

\section{Dissolution test}

The dissolution rate studies on conventional CBZ tablets were conducted according to the USP paddle method at a stirring rate of $75 \mathrm{rpm}$ and a temperature of $37 \pm 0.5 \mathrm{oC}$. The dissolution medium was $900 \mathrm{ml}$ of distilled water containing $0.5 \% \mathrm{w} / \mathrm{v}$ NaLS. The samples were withdrawn at definite time intervals for a total time of 60 minutes and assayed spectrophotometrically at $285 \mathrm{~nm}$. The percentages of cumulative CBZ amounts released from the tablets were calculated. Then data were plotted and compared. ${ }^{6}$

\section{RESULTS AND DISCUSSION}

\section{Compatibility study}

From the FTIR spectra of the pure CBZ powder and the physical mixtures of the drug in CBZ A, B, C, and D, it was observed that the peak of major functional groups of carbamazepine showed no significant shifting from positions. ${ }^{7}$ This means that the drug is physically stable and compatible with the formula in all the selected brands in the study. The results are shown below in figure 1.

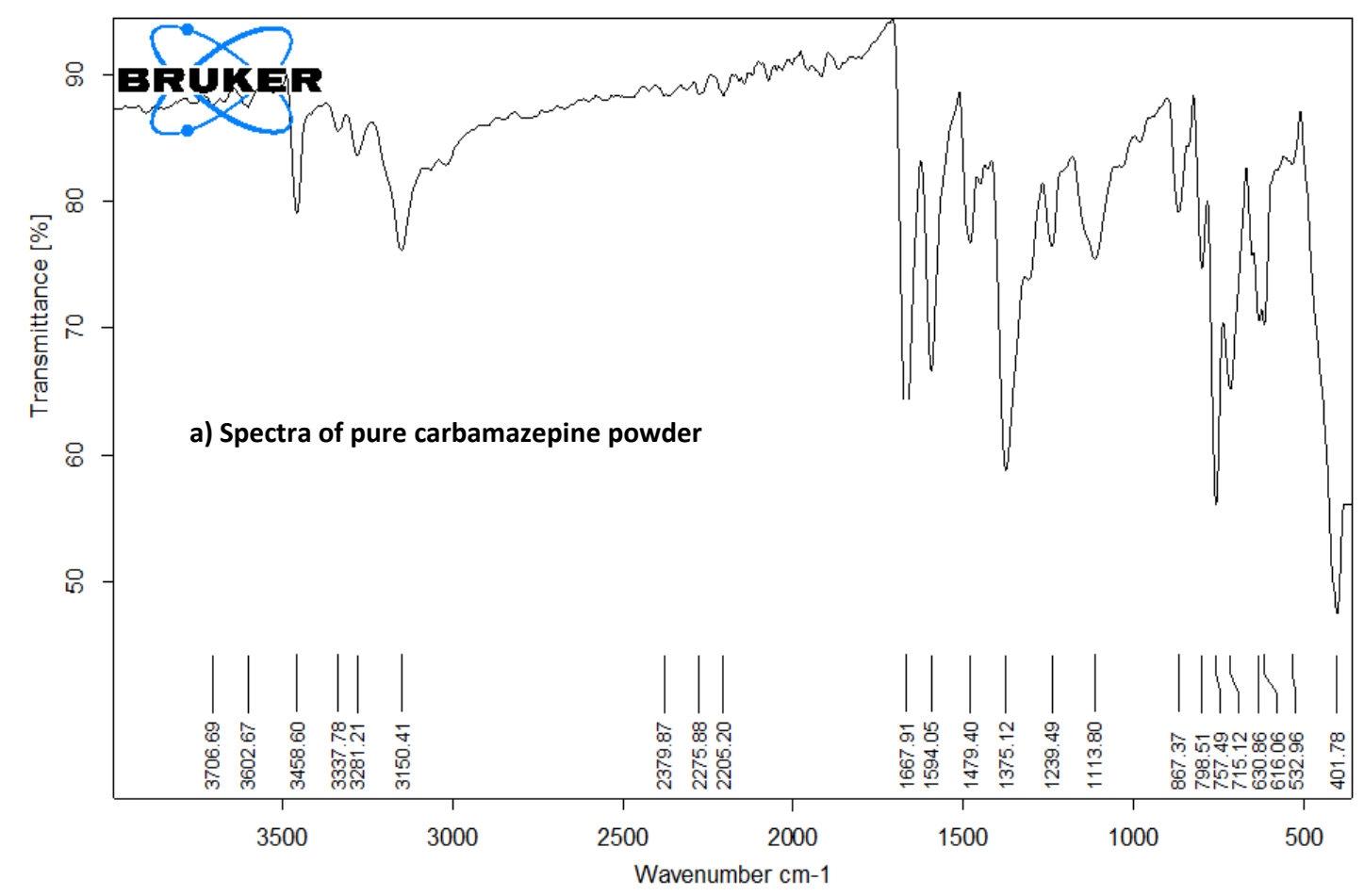



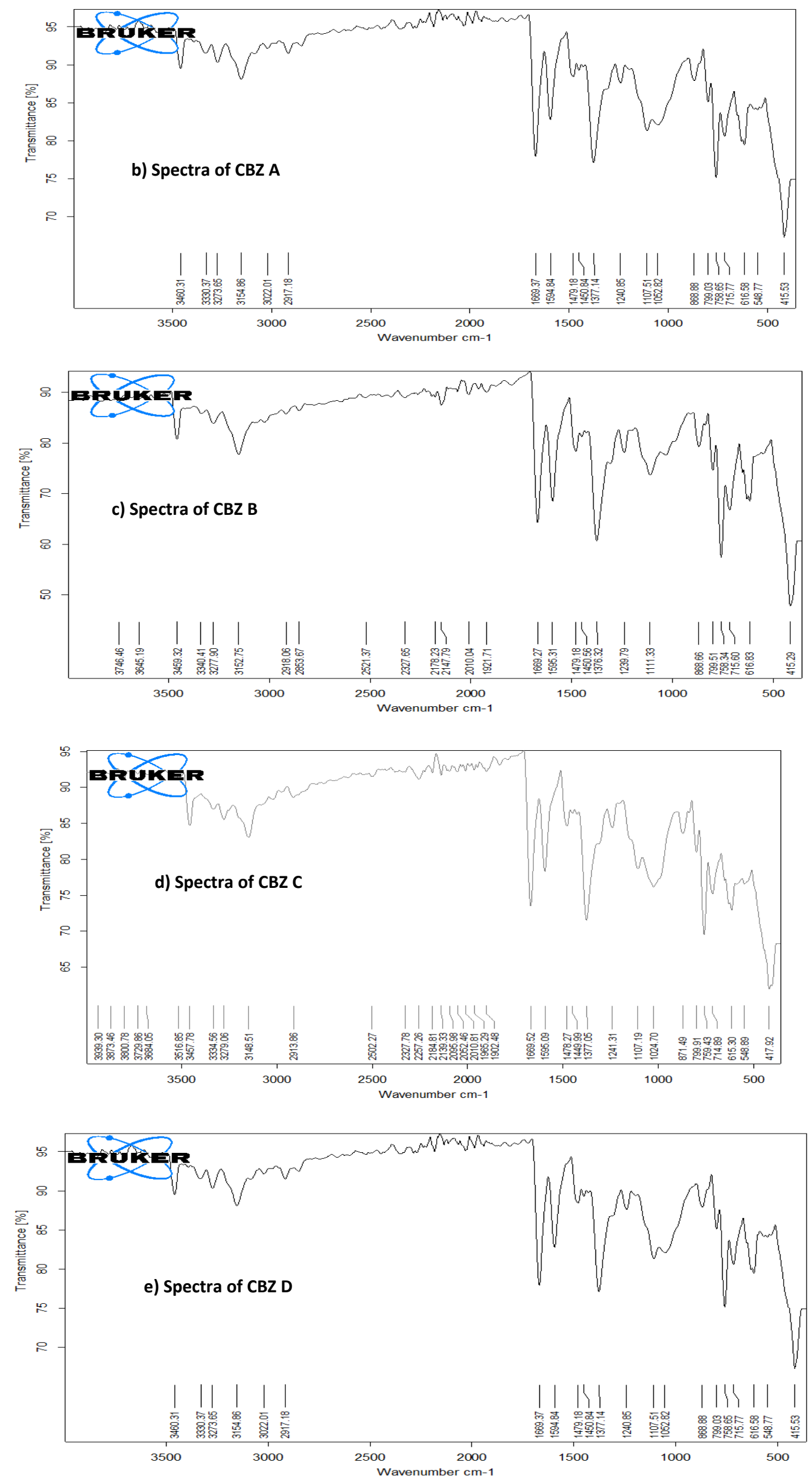

Figure 1: The FTIR spectra of a) pure carbamazepine powder; b) CBZ A; c) CBZ B; d) CBZ C; e) CBZ D 
Calibration curve of carbamazepine

A standard plot of CBZ was plotted as per the procedure in experimental methods and its linearity was shown. The standard graph of carbamazepine shows good linearity with high regression coefficient R2 $=0.998$, which indicates that it obeys beer'sLamberts Law in the concentrations selected. The results are shown in figure (2) below.



Figure 2: The standard calibration curve of carbamazepine raw material in distilled water with $0.5 \%$ sodium lauryl sulphate

\section{Quality control study results}

The results obtained from the quality control tests were given in Table (1) below:

Table 1: Results of quality control tests on four brands of carbamazepine products

\begin{tabular}{|l|l|l|l|l|l|}
\hline $\begin{array}{l}\text { Tablet Generic } \\
\text { Code }\end{array}$ & $\begin{array}{l}\text { Tab. Weight } \\
\text { (average) mg. }\end{array}$ & Hardness (kg) & Friability (\%) & $\begin{array}{l}\text { Drug content } \\
(\%)\end{array}$ & $\begin{array}{l}\text { Disintegration } \\
\text { time (sec) }\end{array}$ \\
\hline (CBZ) A & 465.2 & 6.1 & 0.58 & 99.89 & 191 \\
\hline (CBZ) B & 279.6 & 4.3 & 0.36 & 98.68 & 172 \\
\hline (CBZ) C & 325 & 5.2 & 0.51 & 9.43 & $\begin{array}{l}\text { Failed } \\
\text { Less than } 50\end{array}$ \\
\hline (CBZ) D & 261 & 4.4 & & 93 & 151 \\
\hline
\end{tabular}

All CBZ tablets contain not less than 98.68 percent and not more than 99.89 percent of the labeled amount of active drug (except CBZ D). Content uniformity test results showed that all conventional CBZ tablets fit these criteria, but the product CBZ D was failed to pass this test.

Although there is no official test for hardness, this property must be controlled to ensure that the product is firm enough to withstand handling without breaking or crumbling and not so hard that the disintegration time is unduly prolonged. The recommended value for tablet hardness is $4-8 \mathrm{~kg} .{ }^{7}$ All tablets supply the required hardness limits from $4.3 \mathrm{~kg}-6.1$ $\mathrm{kg}$.

The friability value which is also affected by the hardness value of tablets should be in the range of $0.0-1 \%$ limits. $^{7}$ Friability values of all the tablets used are in the range of $0.36 \%-0.58 \%$, these results indicate that all the brands met the pharmacopeial requirements of tablet formulation.

\section{Dissolution data}

As shown in figure (3) below, CBZ A released almost about $75 \%$ and $96 \%$ of its drug load at the end of the $5^{\text {th }}$ and $20^{\text {th }}$ minutes, respectively. This high release rate may be attributed to the incorporation of tablet excipients that act as release promoters or solubilizers during the manufacturing of CBZ A, which explain the relatively high tablet weight of CBZ A tablet compared to other products (465.5 mg)



Figure 3: The cumulative release profile of CBZ A product

CBZ B and CBZ C, on the other hand, showed very comparable release profiles as shown in figures (4) and (5) below. Both brands release about $75 \%$ of their drug load at the time of 40 minutes and about $85 \%$ of the drug load after 60 minutes. 


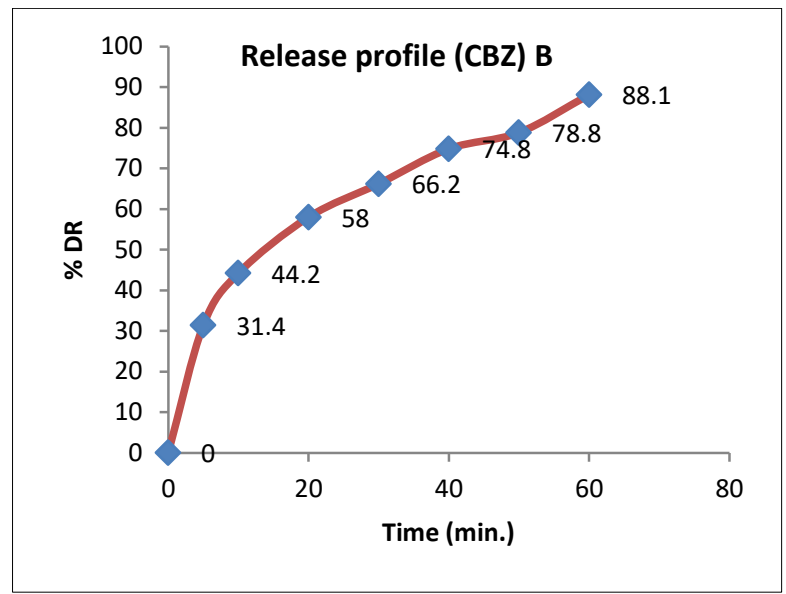

Figure 4: The cumulative release profile of CBZ B product

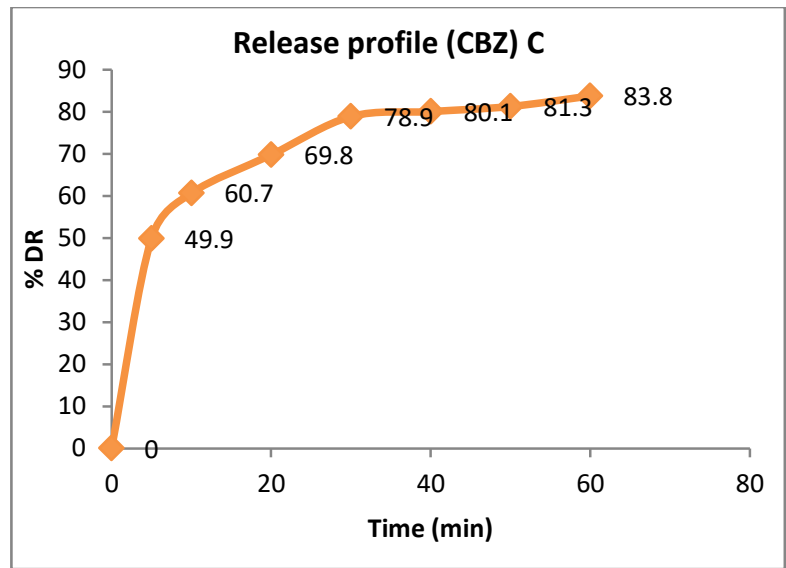

Figure 5: The cumulative release profile of CBZ C product

However, CBZ C showed a burst release about 1.5 times greater than that of CBZ B at the end of the tenth minute (about $60 \%$ of the drug load in CBZ C versus $44 \%$ of the drug load in CBZ B).

This difference in the burst release may represent a therapeutic issue for some patients. ${ }^{8}$

Finally, as shown in figure (6) CBZ D have been failed to provide the required dose of the drug carbamazepine (which is at least $150 \mathrm{mg}$ of the drug at the end of one hour). This failure is not necessarily due to failure of drug release from the dosage form, but it is mainly due to low drug content (less than half of the recommended dose) in the final product that illustrated in table 1.

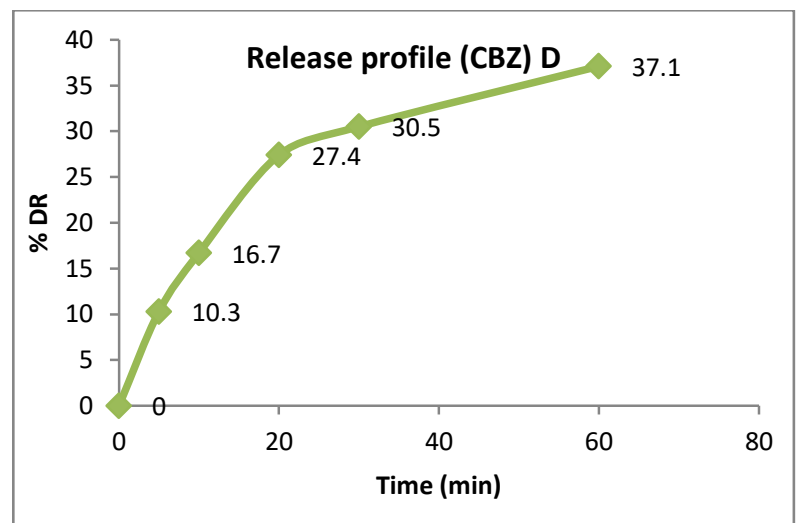

Figure 6: The cumulative release profile of CBZ D product

\section{CONCLUSION}

The primary goal of any dosage form is to release the recommended amount of the active drug in an expected time interval, and according to the USP XXVIII, conventional tablet monographs for class II drugs (CBZ in this study) must release at least $75 \%$ of the labeled amount of the drug at the end of 60 minutes. ${ }^{6}$ As can be seen in figure 7, below, all the brands of conventional CBZ tablets available in the Iraqi pharmaceutical market, except CBZ D, met this requirement.

Consequently, patients stabilized on CBZ A are used to receive a relatively high drug load just a few minutes after tablet ingestion, and they should be advised never to switch their medication to another brand unless a close plasma drug monitoring and therapeutic response monitoring are conducted. ${ }^{9,10}$

CBZ B and CBZ C showed a comparable in-vitro release profile. Therefore, in case of unavailability of each one of them offers an acceptable alternative to the other when its unavailable to use by the patient.

Surprisingly, CBZ D failed to release the recommended amount of the drug load labeled by the manufacturer and thus patients must be advised not to use it at any circumstance, otherwise, the probability of case relapse will be very high. ${ }^{11}$

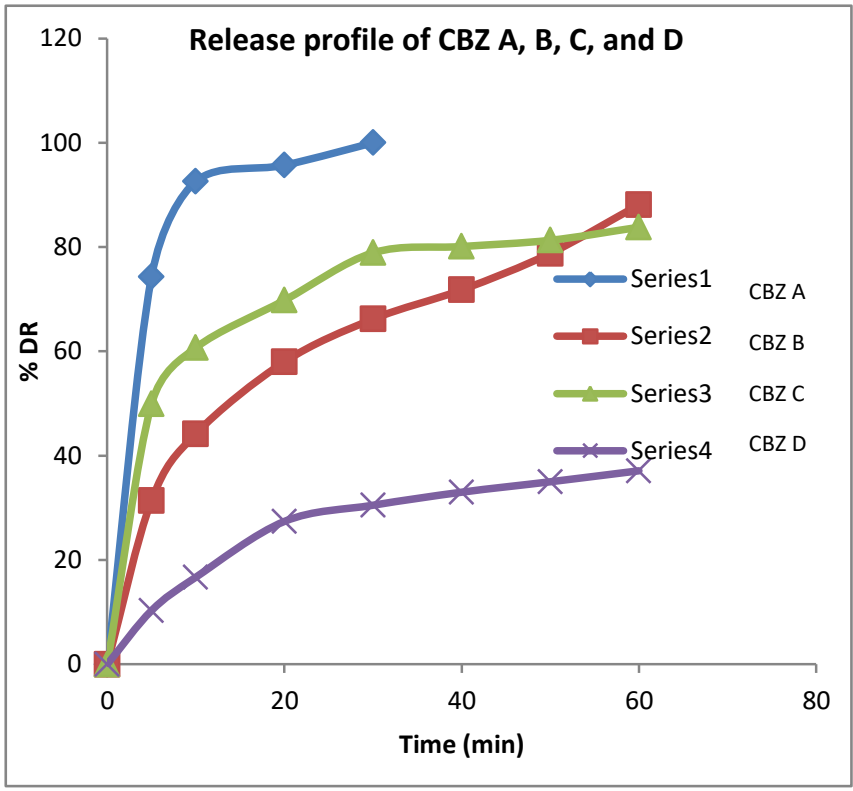

Figure 7: The cumulative release profiles of four brands of carbamazepine tablets

\section{REFERENCES}

1. Organization WH. Quality assurance of pharmaceuticals: A compendium of guidelines and related materials. Good manufacturing practices and inspection: World Health Organization; 2007.

2. Bertilsson L, Tomson T. Clinical pharmacokinetics and pharmacological effects of carbamazepine and carbamazepine10, 11-epoxide. Clinical pharmacokinetics. 1986 Sep; 11(3):17798.

3. Tolou-Ghamari Z, Zare M, Habibabadi JM, Najafi MR. A quick review of carbamazepine pharmacokinetics in epilepsy from 1953 to 2012. Journal of research in medical sciences: the official journal of Isfahan University of Medical Sciences. 2013 Mar; 18(Suppl 1):S81.

4. Oluka MO, Mitema ES, Kibwage IO, Kwasa TO, Kokwaro GO. A comparative bioavailability of four Carbamazepine tablet 
formulations available in the Kenyan market. East African medical journal. 1996 May 1; 73(5):323-6.

5. Kranz H, Wagner T. Effects of formulation and process variables on the release of a weakly basic drug from single unit extended release formulations. European journal of pharmaceutics and biopharmaceutics. 2006 Jan 1; 62(1):70-6.

6. USP. United States Pharmacopeia. United States Pharmacopeial; 2015.

7. Moffat AC, Osselton MD, Widdop B, Watts J. Clarke's analysis of drugs and poisons. London: Pharmaceutical press; 2011.

8. Fortuna A, Alves G, Soares-da-Silva P, Falcão A.

Pharmacokinetics, brain distribution and plasma protein binding of carbamazepine and nine derivatives: new set of data for predictive in silico ADME models. Epilepsy research. 2013 Nov 1; 107(1-2):37-50.

9. Panday DR, Panday KR, Basnet M, Kafle S, Shah B, Rauniar GP. Therapeutic drug monitoring of carbamazepine. Int J Neurorehabilitation Eng. 2017; 4(245):2376-0281.

10. Bertilsson L, Tomson T. Clinical pharmacokinetics and pharmacological effects of carbamazepine and carbamazepine10, 11-epoxide. Clinical pharmacokinetics. 1986 Sep; 11(3):17798.

11. Ayano G. Bipolar disorders and carbamazepine: pharmacokinetics, pharmacodynamics, therapeutic effects and indications of carbamazepine: review of articles. J Neuropsychopharmacol Ment Health. 2016; 1(112):2. 